\title{
La vocation religieuse féminine dans les années 1960-1970 : crise collective, itinéraires singuliers
}

\section{Sabine Rousseau}

\section{(C) OpenEdition \\ Journals}

\section{Édition électronique}

URL : http://journals.openedition.org/abpo/503

DOI : $10.4000 / a b p o .503$

ISBN : 978-2-7535-1515-4

ISSN : 2108-6443

\section{Éditeur}

Presses universitaires de Rennes

Édition imprimée

Date de publication : 30 octobre 2009

Pagination : 125-138

ISBN : 978-2-7535-1008-1

ISSN : 0399-0826

\section{Référence électronique}

Sabine Rousseau, «La vocation religieuse féminine dans les années 1960-1970 : crise collective,

itinéraires singuliers », Annales de Bretagne et des Pays de l'Ouest [En ligne], 116-3 | 2009, mis en ligne le 30 octobre 2011, consulté le 02 mai 2019. URL : http://journals.openedition.org/abpo/503 ; DOI :

$10.4000 / a b p o .503$ 


\title{
La vocation religieuse féminine dans les années 1960-1970 : crise collective, itinéraires singuliers *
}

\author{
Sabine RousSEAU \\ Docteur en histoire contemporaine \\ chargée de cours à l'IEP de Lyon, \\ CHEC - université Blaise-Pascal de Clermont-Ferrand
}

Dans les années 1960 et 1970, la crise des vocations religieuses féminines devient patente et donne lieu à une réflexion sur son origine, à des interrogations sur la vocation elle-même et à l'élaboration de réponses pour y faire face. Comment l'Église catholique la perçoit-elle et cherche-t-elle à y remédier? Quelles sont les conséquences de la crise des vocations et des réponses apportées sur les conceptions de la vocation elle-même considérée ici dans ses deux acceptions, à savoir au sens restreint d'entrée en religion et au sens large d'engagement religieux vécu dans la durée? Pour éclairer ces questions, il faut mettre en perspective la crise des vocations religieuses féminines avec celle des vocations sacerdotales, en décrire la réalité ainsi que les perceptions qu'on en a dans l'Église avant de présenter les premières réponses, pastorales, apportées avant Vatican II. Ensuite on pourra analyser les autres réponses élaborées dans le sillage du concile pour palier les insuffisances des premières : des réponses ecclésiales en faveur de la « rénovation de la vie religieuse " mais aussi des réponses théologiques.

Faire l'histoire de la vocation ou ici de la crise des vocations, c'est tenter une histoire collective d'un engagement personnel ce qui implique de se placer à deux échelles différentes, de croiser les réponses institutionnelles à la crise et des itinéraires singuliers. Celui de Françoise Vandermeersch (1917-1997), directrice de la revue de la congrégation des Auxiliatrices, Échanges, très impliquée dans l'élaboration des réponses pastorales puis ecclésiales à la crise des vocations religieuses féminines fournit un exemple riche.

* Cet article est issu d'une communication orale présentée en avril 2007 à l'université du Maine lors d'une journée d'étude organisée par Hervé Guillemain et consacrée aux vocations religieuses et laïques. 


\section{Crise des vocations : réalités et perceptions}

\section{Crise du recrutement...}

La crise des vocations religieuses féminines apparaît dans un contexte plus global de crise des vocations sacerdotales. Les travaux de Martine Sevegrand ${ }^{1}$ ont montré que si l'on considère le nombre annuel d'ordinations dans chaque diocèse, la crise de la vocation sacerdotale est précoce : elle se fait sentir dès la fin des années 1940. Après 1952, on ne dépasse plus jamais les 1000 ordinations par an, dès 1967 on tombe à moins de 500 et après 1974, à moins de 200. Dans les années 1960, la question de " la crise du sacerdoce " est un lieu commun récurent, qui a déjà fait le titre d'un ouvrage du jésuite Paul Doncour en 1932. La réflexion s'est amorcée sur la mission du prêtre avec le livre des abbés Godin et Daniel, La France pays de mission?, paru en 1943. Depuis l'après-guerre, le malaise du clergé est perçu par les évêques attentifs au déclin des vocations. Ils alertent l'opinion chrétienne car ils estiment que la crise provient de la réticence des familles chrétiennes à orienter leurs fils vers la prêtrise; ils incriminent aussi le matérialisme qui détourne les jeunes de la vie spirituelle et expliquent ainsi le tarissement des vocations par la résistance à la grâce.

La crise de vocation des religieuses est plus discrète : on commence seulement à en parler dans les années 1960 et surtout dans les années 1970. Une enquête lancée par l'Union des Supérieures majeures de France en 1969 met en évidence la baisse des effectifs ${ }^{2}$. En 25 ans, depuis la fin de la Seconde Guerre mondiale, la baisse des effectifs a atteint $13 \%$. En février 1969 vivaient en France 114428 religieuses dont plus de 104000 de vie apostolique. La moyenne d'âge était alors de 55 ans. En 1977, les apostoliques n'étaient déjà plus que $92000^{3}$. Cette baisse des effectifs est imputée à une crise du recrutement, sensible dès 1933-1937 mais qui s'est accentuée à partir de 1960 et surtout à partir de 1965. Les raisons évoquées ne diffèrent pas de celles avancées pour expliquer la crise des vocations sacerdotales : les tendances hédonistes de la société, des comportements individualistes voire égoïstes, détourneraient les jeunes filles d'un engagement spirituel profond. La version féminine de la crise de recrutement est reconnue plus tardivement mais analysée selon la même grille que celle des vocations sacerdotales, de loin plus préoccupante aux yeux des autorités, inquiètes de l'avenir des paroisses.

Avant Vatican II, les premières réponses apportées par l'institution sont fondées sur ces interprétations de la crise. Le fait d'imputer cette crise des vocations à des facteurs externes à l'Église implique des réponses institutionnelles d'ordre essentiellement pastoral. Une littérature

1. SEVEGRAND, Martine, Vers une Église sans prêtres, la crise du clergé séculier en France (1945-1978), Rennes, PUR, 2004.

2. Enquête P. Luchin pour l'Union des Supérieures Majeures de France, février 1969.

3. HourTicQ, Christiane, Les Religieuses, Paris, Éditions de l'Atelier, 1996. 
pratique, " une littérature de recrutement ${ }^{4}$ ", est diffusée auprès de ceux chargés de la direction de conscience, de discerner et de cultiver les " germes de la vocation ". Il s'agit de permettre une plus grande efficacité " technique " dans l'art de conduire le recrutement des prêtres essentiellement mais aussi des religieux et des religieuses. À cet effet, en 1959, est créé le Centre national des vocations pour favoriser la recherche et l'accompagnement de nouvelles vocations sacerdotales et religieuses grâce à un travail à la fois doctrinal et pastoral. Françoise Vandermeerch y participe comme conseillère car elle a acquis des compétences par l'accueil, dans son couvent de la rue du Cherche-Midi à Paris, de jeunes retraitantes auxquelles elle propose les exercices spirituels de type ignacien. Depuis 1954, elle encourage et cultive le discernement personnel chez des jeunes filles qui, hésitant parfois entre mariage et vie religieuse, cherchent leur vocation. Entre 1954 et 1966, une cinquantaine d'entre elles a opté pour la vie religieuse à la suite d'une retraite sous la direction spirituelle de Françoise Vandermeersch.

En 1964, la hiérarchie apporte une autre réponse pastorale : Paul VI institue une journée mondiale des vocations pour tenter de redonner de l'attrait à l'engagement religieux. C'est la généralisation d'initiatives locales qui avaient vu le jour dans certains diocèses où chaque année une journée des vocations était organisée. Dans son enquête sur le recrutement des séminaristes en Vendée entre 1945 et le début des années 1970, Charles Suaud a pu montrer les dispositions prises par le clergé local pour favoriser le recrutement de nouveaux prêtres : il voit dans ce dispositif une " théologie pratique " destinée à repérer les signes de la vocation dès le début de l'adolescence. Il y lit des critères de recrutement fondés sur une bonne santé, des aptitudes intellectuelles, des qualités morales, un bon caractère, un goût certain pour la piété, un environnement familial chrétien honorable et le désir d'être prêtre. Dans l'arsenal de détection des vocations, la retraite apparaît comme " l'ultime instrument de marquage ${ }^{5}$ ".

\section{...Ou crise de la vie religieuse?}

La hiérarchie catholique admet tardivement, au cours des années 1960 que le malaise du clergé est plus profond puisqu'il se traduit aussi par des défections : le nombre de départs de prêtres séculiers double entre la période 1960-1964 (autour de 240 en 5 ans) et la période 1965-1969 (485 en 5 ans) et double à nouveau en 1970-1974 (autour de 970 en 5 ans) avec un pic en 1972. En acceptant d'admettre que la réduction à l'état laïc puisse dispenser du célibat, l'Église catholique reconnaît implicitement, en 1964, la réalité des défections.

4. Expression de Louis Dingemans, dans « La Vocation, appel de Dieu, phénomène d'Église ", Cahiers de Froidmont, 20, 1976, p. 9.

5. SuAud, Charles, La Vocation, conversion et reconversion des prêtres ruraux, Paris, Éditions de Minuit, 1978. 
En ce qui concerne les religieuses, il est plus difficile encore de l'admettre. En janvier 1970, dans deux articles du journal Le Monde, Robert Solé, signalant une vague de départs aux États-Unis, aux Pays-Bas, en Belgique, affirme qu'en France les congrégations, seules en possession de chiffres, ne souhaitent pas les faire connaître ${ }^{6}$. Il estime alors qu'un millier de religieuses-professes ont quitté chaque année le couvent dans la deuxième moitié des années soixante. D'autres chiffres donnent 350 départs par an entre 1969 et 1972 contre 150 dans la période 1945-1968 ${ }^{7}$.

Françoise Vandermeersch est une des rares religieuses à faire en sorte que la question de la crise de la vocation ne soit pas niée mais analysée dans sa totalité, à savoir non seulement en termes de crise de recrutement mais qu'il soit fait également mention des départs qui affectent les congrégations dans les années 1960. En effet, considérant que les départs après engagement définitif ne sont pas des faits isolés, Françoise Vandermeersch consacre deux numéros de sa revue Échanges ${ }^{8}$ en 1968 et 1969 à poser le problème de fond de la vocation. Pour elle, il ne s'agit pas seulement de défections individuelles dues à des crises personnelles mais d'un phénomène général :

"La crise des vocations dont on parle souvent ne peut se comprendre dans le sens d'appels de Dieu qui se feraient plus rares ni même de réponses moins généreuses. Elle se situe plutôt dans l'inadaptation des formes actuelles en regard des désirs et des capacités de ceux et celles qui aspirent à se consacrer à Dieu ${ }^{9}$."

Elle met en cause la formation des novices, mal préparées, selon elle, à faire face aux problèmes nés du célibat et du renoncement à la maternité. Dans un bref article, qu'elle signe d'un pseudonyme, dans le numéro d'Échanges de juin 1968, elle pose la question de la pertinence du célibat ${ }^{10}$. Elle fait plusieurs conférences sur le thème des " problèmes de l'affectivité des religieuses " dans lesquelles elle aborde le sujet tabou de la sexualité ${ }^{11}$. Mais Françoise Vandermeersch estime surtout que la vague de départs révèle plus largement l'inadéquation du mode de vie religieux avec la société moderne et l'écart grandissant entre l'évolution de la condition féminine vers l'émancipation et le "fixisme " du statut des religieuses dans l’Église. Depuis 1967, elle ose décrire les aspects « archaïques » du quotidien

6. "Ces religieuses qui quittent le couvent ", Le Monde, 11 et 12 janvier 1970, cité dans Potel, Julien, "Portes ouvertes chez les religieuses ", Paris, L'Harmattan, 1999, p. 208.

7. Michelat, Guy, Potel, Julien, Martre, Jacques, Les Français sont-ils encore catholiques? Paris, Cerf, 1992, p. 43 cité dans Potel, ibid. Les Dossiers de l'Union des Supérieures majeures datés de mai-juin 1971 ont conservé le chiffre de 150 départs par an.

8. "La vocation religieuse féminine ", Échanges, n ${ }^{\circ} 86$, juin 1968 et "Religieuses, un scandale ", Échanges, n' $93,1969$.

9. Éditorial, Échanges, ${ }^{\circ} 86$.

10. "Le Célibat contesté ", extrait d'une conférence aux séminaristes, Issy-lesMoulineaux, février 1968, Échanges, n 86 , p. 29.

11. Par exemple, conférence le 28 janvier 1971 au Centre Saint-Louis des Français à Rome, Archives Françoise VANDERMEERSCH (Arch. FV) 
des religieuses, des " prescriptions d'un autre âge ", du port obligatoire du bonnet de nuit à la surveillance du courrier personnel ${ }^{12}$... Elle estime qu'il faut viser " le plein épanouissement de la personnalité humaine et féminine des religieuses $"{ }^{13}$. Pour cela, elle appelle de ses voeux une reconnaissance de la personnalité des religieuses, de leur autonomie. Elle plaide pour un plus grand respect de leur intimité. Elle exprime le souhait que toutes puissent accéder à un espace privé leur garantissant une chambre individuelle, le secret de la correspondance, l'assurance de pouvoir entretenir des liens familiaux et amicaux. Elle parle de l'équilibre du corps à atteindre grâce à une plus grande attention à soi : repos, loisirs, hygiène et soin du corps doivent entrer dans le quotidien des couvents.

Cette prise de parole, rare chez les religieuses, se situe alors dans la dynamique des textes conciliaires, en particulier du décret Perfectae caritatis qui a servi à Françoise Vandermeersch de base pour faire avancer la réflexion sur la nécessité de moderniser les conditions de vie des religieuses.

\section{Réponses ecclésiales et expériences communautaires \\ Perfectae Caricatis et la réforme de rénovation de la vie religieuse}

Devant l'insuffisance des réponses de type pastoral pour allumer de nouvelles vocations, les pères du Concile ont été amenés à s'interroger plus avant sur la vie religieuse, sur la vocation au sens large, considérée ici comme un engagement dans la durée. C'est au cours de la IV session de Vatican II, que le décret sur la rénovation de la vie religieuse est voté. Ce texte met fin au primat du modèle de la perfection par le renoncement au monde et met l'accent sur la mission apostolique des religieux et religieuses. S'inscrivant dans une volonté générale d'ouverture sur le monde, il accorde une place plus grande à l'épanouissement personnel de ceux et celles qui ont choisi la vie communautaire. Il incite les instituts à procéder eux-mêmes à une triple rénovation : celle des relations des communautés avec " le monde", celle des rapports des membres des congrégations entre eux et celle des pratiques religieuses individuelles, notamment spirituelles. Il appelle à inaugurer des expériences nouvelles pour répondre aux " exigences " du temps : en acceptant de se pencher sur les (dys)fonctionnements internes des instituts, il constitue une forme de réponse au "malaise " qui se traduit notamment par la vague de départs à laquelle l'Église est confrontée. Il est demandé aux congrégations de réviser leurs statuts avant 1970.

Un des deux numéros d'Échanges sur les religieuses paraît au moment où des décrets expérimentaux sont rédigés chez les Auxiliatrices au cours d'un débat d'un mois et demi en octobre 1969. Ces constitutions provisoires mettent l'accent sur l'autonomie des religieuses dans la vie apostolique, sur la participation et le dialogue pratiqués dans un esprit collégial quant au

12. Françoise VANDERMEERSCH, Commentaire de Perfectae caritatis, 1967, Arch. FV.

13. Ibidem, p. 9. 
mode de gouvernement de la congrégation et traduisent l'aspiration des religieuses à davantage de respect de leur personnalité et de leur intimité y compris dans des aspects très matériels de la vie quotidienne évoqués par Françoise Vandermeersch.

Cependant il s'agit davantage d'ajustements que de remise en cause. Certes, la liberté de parole va de pair avec la reconnaissance de l'individu mais au fond les piliers de la vie religieuse sont réaffirmés : pauvreté (" rebaptisée " partage), obéissance (devenue " participation " et " dialogue ") et chasteté (à laquelle est préférée l'expression " célibat religieux ") demeurent les fondements de l'engagement religieux. La congrégation demeure l'institution de référence. Or, à la fin des années 1960 et au début des années 1970, des religieuses, comme Françoise Vandermeersch, expriment le besoin d'un renouvellement plus profond du modèle de la vie religieuse et cherchent à inventer de nouvelles formes de vie apostolique. Les réponses ecclésiales données à la crise des vocations religieuses basées sur une rénovation du vécu des religieuses dans leur communauté ne leur paraissent pas suffisantes. Certaines religieuses se lancent alors dans des expériences nouvelles : celles des petites communautés.

\section{L'expérience des petites communautés}

Dans un contexte qui voit naître des "communautés de base ${ }^{14}$ ", Françoise Vandermeersch écrit au chancelier de l'Archevêché de Paris, en janvier 1971, pour lui demander un local afin d'héberger une petite communauté qui serait composée de six à sept personnes : des Auxiliatrices qui ont reçu l'accord de leurs supérieures et deux jeunes filles qui désirent s'orienter vers une vie religieuse apostolique. Par cette démarche, Françoise Vandermeersch tente de concrétiser son idée de renouvellement des formes de la vie religieuse en promouvant des "petites communautés nouvelles ", " souples et ouvertes ", composées à la fois de religieuses ayant prononcé des vœux définitifs et des laïques désirant expérimenter une " vie communautaire évangélique " dans laquelle elles pourraient " s'engager pour un temps limité ou illimité, sans obligation de vœux perpétuels, la forme de l'engagement étant laissée à la liberté de chacun ${ }^{15}$ ". Françoise Vandermeersch considère en effet que le renouvellement passe par " une double recherche, déjà commencée par les religieuses " mais pour laquelle elle juge « souhaitable qu'elle se fasse aussi par celles qui, appelées à partager leur vie, en récusent les conditions actuelles ${ }^{16}$ ". Dans sa lettre à l'Archevêché, elle explique que toutes souhaitent " renouveler la tradition de leur congrégation " car elles croient " à la vie religieuse à condition de la rendre plus accessible aux jeunes ". Elle conclut : "En définitif, nous

14. BeSREt, Bernard, Schreiner, Bernard, Les Communautés de base, Grasset, 1973.

15. VANDERMEERSCH, Françoise, "La Vie religieuse en question ", dans "Réinventer l'Église? ", Esprit, novembre 1971, p. 733-734.

16. Éditorial d'Échanges, ${ }^{\circ} 86$, juin 1968. 
cherchons à réaliser un témoignage évangélique désencombré d'un certain poids des formes passées ${ }^{17}$ ". Elles veulent donc créer un lieu " d'accueil " (pour recevoir des " amis, catéchumènes, relations diverses ${ }^{18}$ "), un lieu de " communication ", de " rencontre ", de " partage ", un lieu de " cristallisation ", " de recherche et d'expérimentation ${ }^{19}$ "; un lieu " à la fois émancipé par rapport aux organisations officielles et en situation de solidarité voulue avec elles ». Françoise Vandermeersch insiste sur le maintien de la "référence à une famille religieuse et à une tradition spirituelle ", ici ignacienne. Dans un numéro des Dossiers de l'Union des Supérieures majeures, en maijuin 1971, Françoise Vandermeersch appelle de ses vœux la généralisation de structures souples, l'établissement de " communautés plus réduites, dépendantes bien sûr d'une responsable mais sans l'obligation de passer par tous les degrés de la hiérarchie ${ }^{20}$ ".

Ces projets vont au delà de la simple rénovation de la vie religieuse à l'intérieur des maisons religieuses proposée par l'institution ecclésiale mais ambitionne de renouveler « les formes de la présence de l'Église ». On assiste là à une mise en question plus profonde de la nature même de la vie religieuse communautaire.

\section{Rééquilibrages théologiques et révisions personnelles Rééquilibrages théologiques}

Ces réflexions sur la rénovation de la vie religieuse ou plus avant sur le renouvellement des formes de la vie religieuse s'accompagnent aussi d'une recherche théologique sur la vocation elle-même, au sens étroit, qui conduit à un rééquilibrage entre les trois " acteurs " traditionnellement considérés comme étant à l'œuvre dans la naissance et l'épanouissement d'une vocation : l'appelant, l'appelé et l'Église. L'équilibre entre ces acteurs a fait l'objet d'âpres débats notamment au début du Xx $x^{\mathrm{e}}$ siècle lors de la controverse Branchereau/Lahitton ${ }^{21}$. À une " théorie de l'attrait " privilégiant le désir et les signes personnels et subjectifs de la vocation ${ }^{22}$, s'oppose une théorie de l'appel de l'Église, seule habilitée à reconnaître la véritable vocation et à la légitimer, privilégiant ainsi le rôle de la hiérarchie dans l'appel au sacerdoce ${ }^{23}$. Cette controverse marquée par le contexte de la crise moderniste ${ }^{24}$, court ensuite le long du siècle : en 1949, elle est relancée par le père Feret dans un

17. Lettre de Françoise VANDERMEERSCH à M. Le Chancelier de l'Archevêché, 6 janvier 1971, Arch. FV.

18. Ibidem.

19. Texte de Françoise VANDERMEERSCH daté du 28 mai 1971 présentant le projet de communauté, Arch. FV.

20. Dossiers de l'Union des Supérieures Majeures, mai-juin 1971, p. 13- 25.

21. Cf. contribution de Gérard LEFEUVRE dans ce volume.

22. BRANCHEREAU, Louis, De la vocation sacerdotale, 1896.

23. LAHITTON, Joseph, La Vocation sacerdotale, 1909.

24. Mettre l'accent sur le rôle de l'évêque permet d'écarter du sacerdoce des séminaristes indésirables. 
article de La Vie spirituelle dans lequel il s'insurge contre la confusion entre le désir et l'appel ${ }^{25}$. Dans l'encyclique Sedes Sapientiae en 1956, Pie XII tranche le débat en parlant d'un double appel, celui de Dieu et celui de l'Église et en réaffirmant l'importance de l'élément divin préalablement à toute vocation. D'où l'importance pour le clergé de savoir discerner les signes de la vocation grâce à une pastorale des vocations allant dans ce sens.

Avec Vatican II, l'appel de Dieu reste essentiel mais on insiste davantage sur la liberté de consentement de l'homme qui adhère au projet, qui s'engage en toute responsabilité dans une expérience spirituelle unique, dans un choix personnel irréductible. La volonté personnelle est valorisée. L'Église est amenée à jouer surtout un rôle de médiation en indiquant et en définissant la mission liée à la vocation sur le chemin choisi à la suite du Christ ${ }^{26}$. La subjectivisation de la vocation peut sembler renforcée au détriment du magistère de l'Église ${ }^{27}$.

Cette tendance générale est bien visible dans la comparaison attentive de deux éditoriaux de Françoise Vandermeersch publiés dans Échanges à dix ans d'intervalle, en 1958 et en $1968^{28}$. En 1958, elle présente longuement l'appel de Dieu, rappelant qu' “ aux premières pages de la Genèse, l'Éternel appelle Abraham " $(1.6,1958)$ puis à propos des Apôtres, que « l'Évangile à son tour, nous rapporte le choix de Dieu : Jésus les appela; sur-le-champ, ils laissèrent leur barque et leur père et ils le suivirent " (1. 9-10, 1958). Ce n'est qu'au milieu de l'éditorial qu'elle commence à parler de la réponse de l'homme : "Cette vocation spéciale, qui est toujours un choix de Dieu, sollicite la réponse de l'homme " (1. 17-18, 1958) alors que dix ans plus tard, elle met d'emblée sur un pied d'égalité l'appel de Dieu et la réponse de l'homme, devenus indissociables : "Transcendance divine, liberté de l'homme se lient de façon mystérieuse et indiscernable dans l'appel et la réponse qui constituent une vocation religieuse " (1. 1-2, 1968). Ensuite seulement elle fait référence aux " choix de Dieu » dans la Bible. Ainsi elle valorise " la réponse de l'homme " dans la vocation en l'érigeant en condition essentielle car dit-elle, " elle réclame l'adhésion de tout l'être " $(1.5,1968)$. Elle utilise pour cela des mots nouveaux, absent de son vocabulaire en 1958 : « liberté de l'homme » $(1.1,1968)$, " conscience » $(1.4,1968)^{29}$. Sur le rôle de l'Église, elle modifie aussi le vocabulaire : elle ne dit plus que l'Église "révèle le sens de l'appel du Seigneur" (1. 13-14, 1958) mais qu'elle " indique le sens spécifique de cet état [religieux] " $(1.25-26,1968)$ valorisant son rôle

25. Cité par LEGRAND, Hervé, "La Théologie de la vocation aux ministères ordonnés : vocation ou appel? ", La Vie spirituelle, décembre 1998, p. 621-640.

26. Sauvage, Michel, "Vocation ", Dictionnaire de spiritualité, Paris, Beauchesne, 1994, t. XVI, col. 1092-1158.

27. C'est le point de vue développé par Hervé LEGRAND dans La Vie spirituelle, 1996, art. cité.

28. Échanges $\mathrm{n}^{\circ} 38$, octobre 1958 et $\mathrm{n}^{\circ} 86$, juin 1968. Cf. textes des éditoriaux en annexe.

29. "La Vocation éclate dans une vie, plus fréquemment elle sourd de la conscience ", 1. 3-4, 1968. 
de médiatrice davantage que celui d'initiatrice. Sur le sens de cette mission, Françoise Vandermeersch ajoute un complément du nom essentiel : elle ne parle plus d'" appel à la perfection " $(1.20,1958)$ mais d'une recherche de la " perfection de l'Amour " (1. 22, 1968) que l'état religieux n'est d'ailleurs pas le seul capable d'accomplir. Les religieuses ne sont plus seulement, comme les Apôtres, " témoins du Christ au milieu du monde " (1. 11-12, 1958 et l. 13-14, 1968) mais " portent témoignage de l'amour du Christ pour les hommes " $(1.35,1968)$. Dans l'épanouissement de sa vocation particulière, la religieuse est utile par ce qu'elle fait davantage que par ce qu'elle est. La mission, ajoute-t-elle, peut même porter " contestation de ce qui dans le monde est contraire à l'Évangile " et non pas " contestation du monde en temps que tel » (1. 33-34, 1968). La mission est devenue partie essentielle de la vocation et la religieuse actrice de sa vocation, qu'elle souhaite ancrer dans sa dimension humaine par une présence accrue au monde.

\section{Révisions personnelles : réécrire son récit de vocation}

Pour Françoise Vandermeersch, les ajustements théologiques s'accompagnent de révisions personnelles, de retour sur son propre itinéraire, de réécriture de son récit de vocation. On dispose pour elle de deux récits de vocation; l'un date de 1969 : c'est le script d'une émission de télévision animée par Pierre Dumayet, "Vocations ${ }^{30}$ "; le second date de 1975 : il s'agit d'un récit autobiographique destiné à être publié ${ }^{31}$. Entre les deux récits séparés de seulement six ans, des différences notables apparaissent, traduisant une évolution rapide de Françoise Vandermeersch.

En 1969, elle parle d'appel intérieur qu'elle dit avoir ressenti la première fois vers l'âge de 10-11 ans et qu'elle aurait fait taire pendant son adolescence. Elle décrit alors cet appel en ces termes : " La vocation religieuse m'est apparue comme absolue, un don radical à la personne de Jésus-Christ qui allait me conduire à Dieu. " Elle raconte ensuite ce qui lui a fait franchir le pas à 19 ans, l'annonce de l'entrée en religion d'une de ses amies :

" Je me souviens très bien du jour où c'est venu, je suis rentrée le soir chez moi, j'habitais la campagne et j'ai pris l'autobus, un peu comme un automate. Je ne savais plus trop ce que je faisais. Je suis arrivée chez moi, je me suis couchée, j'ai prétexté un malaise et puis vraiment tout mon système de valeurs était renversé. J'ai vraiment été bouleversée. Et puis j’ai attendu que ça passe, en faisant le gros dos! et puis ça n'est pas passé, alors je me suis dit : il faut regarder plus près et si Dieu est vraiment Dieu je n'ai pas le droit de rejeter un appel que j'ai ressenti et qui est revenu régulièrement mais que j'ai un peu enterré, je n'ai pas le droit de le laisser passer comme ça, il faut que je voie ce qu'il en est, la volonté de Dieu compte avant tout et par dessus tous les amours humains et tous les projets. Alors j'ai pris mon courage à deux mains, et puis, trois ou quatre mois après j'ai été trouver un prêtre pour lui parler de ma vocation ce qui me coûtait beaucoup parce que

30. Archives FV.

31. VANDERMEERSCH, Françoise, La Vie en face, Paris, Stock, 1976. 
c'était révéler quelque chose justement d'inexprimable, de très intime, et il a pris ça avec beaucoup de sagesse. "

En 1975, Françoise Vandermeersch ne parle plus du tout d'appel : dans cette deuxième version, elle évoque à peine, en une ligne, l'épisode de l'entrée en religion de son amie qui a perdu son rôle d'événement fondateur. Elle présente sa vocation comme une "exigence ", et même comme un choix raisonné effectué dans un contexte historique : le Front populaire. Elle raconte que c'est la confrontation entre la lecture de la Bible, pratiquée depuis l'âge de 15 ans pour, dit-elle, " chercher un sens à la vie " et les grèves de 1936 à l'usine de son père, patron d'une usine textile dans le Nord de la France, qui a fait naître sa vocation. " J'étais saisie alors par un immense désir de changer le monde; mais comment faire? C'est alors qu'en ruminant l'Évangile, et aussi les textes vibrants des prophètes de l'Ancien Testament, je commençai à me dire qu'en acceptant de tout laisser, famille, maison, confort, pour partager avec d'autres une vie de pauvreté et de service dans la vie religieuse, je participerais un peu au changement. Car pour moi à cette époque, il était impensable d'envisager un engagement politique. L'engagement dans la vie religieuse restait donc la seule voie ouverte à ma soif de justice ${ }^{32}$. " Elle tend donc à montrer que sa vocation résulte de la mise en adéquation d'un projet politique, au sens large, à sa position sociale de femme issue de la bourgeoisie catholique.

Les différences fondamentales apparaissent : le second récit ne relate plus un " appel indescriptible " mais une " exigence " qui débouche sur un choix rationnel. Ce récit est ancré dans une réalité socio-politique et ne fait plus référence à un événement fondateur, une révélation personnelle. Il ne se fixe plus sur l'entrée en religion mais insiste sur la mission elle-même. Entre 1969 et 1975, on assiste à une forme de sécularisation de ce récit de vocation qui correspond à une évolution de la position sociale personnelle de Françoise Vandermeersch. En effet, au début des années 1970, elle a été amenée à prendre des distances avec sa congrégation : après l'échec de son projet de petite communauté, après les avertissements reçus de la hiérarchie à propos de ses prises de position sur des questions morales (la contraception et l'avortement) ou ecclésiales (la question du sacerdoce des femmes ou du mariage des prêtres), elle s'est installée seule dans les locaux de sa revue désormais détachée de la congrégation et gérée par une association de laïcs. Bientôt, Françoise Vandermeersch devient présidente et principale animatrice d'une autre association laïque à caractère humanitaire, Fraternité chrétienne avec le Vietnam, à laquelle elle consacre les vingt dernières années de sa vie, tout en restant fidèle au statut social que sa vocation initiale de religieuse lui confère.

Les événements de la décennie 1965-1975 ont conduit Françoise Vandermeersch à penser et à vivre différemment sa vocation, à la faire

32. La Vie en face, op. cit., p. 73-74. 
glisser vers une vocation militante qui emprunte à la vocation religieuse un certain nombre de ses traits, notamment le dévouement à une cause et le service aux autres par l'abnégation de soi induite par une disponibilité totale. Elle vit cette forme nouvelle d'engagement dans la continuité, comme un renouvellement de sa vocation, à un moment où il lui est devenu indispensable de trouver un nouveau modus vivendi avec l'institution qui lui permette de vivre, dans l'indépendance, sa vocation religieuse dans une vocation militante. À l'heure des départs nombreux, Françoise Vandermeersch a construit un itinéraire singulier dans l'Église en guise de réponse individuelle à la crise des vocations féminines à laquelle l'institution n'a pas apporté de réponse suffisamment audacieuse pour inventer de nouvelles formes d'engagement religieux féminin.

Dans cette première moitié des années 1970, des " chrétiens en recherche » tentent pourtant de faire évoluer la théologie de la vocation : la question de l'accès au ministère ordonné des hommes mariés et des femmes anime de nombreuses réunions et fait couler beaucoup d'encre ${ }^{33}$. Bien qu'il alimente aussi la réflexion sur la place des femmes dans l'Église, ce débat focalise néanmoins l'attention sur la crise des vocations sacerdotales masculines... au détriment de celle des vocations religieuses féminines.

33. « La Vocation, appel de Dieu, phénomène d'Église », Cahiers de Froidmont, 20, 1976. Il s'agit d'un bilan des recherches sur la vocation à la lumière des apports des sciences humaines, en particulier de la sociologie, bourdieusienne notamment, et de la psychologie, dans une démarche d'historicisation de la vocation, effectué entre 1974 et 1976 par une équipe de dominicains belges ayant organisé une dizaine de réunions avec des professeurs des différentes universités catholiques et protestantes et de Séminaires en Belgique. Cf. aussi les travaux du groupe Femmes et Hommes dans l'Église (FHE) né en 1971, en Belgique également. 


\section{Annexe 1 - Éditorial d'Échanges, $n^{\circ} 38$, oct. 1958.}

Quitte ton pays, ta famille, la maison de ton père, et va vers le pays que je te montrerai (Gen., XII, 1).

LA VOCATION fondamentale de l'homme est un échange d'amour, un partage d'amour avec Dieu même. Mais, dans cette destinée universelle, il y a des vocations spéciales : l'élu est alors choisi par Dieu, réservé pour le service du peuple de Dieu, et ce choix même est marqué par l'amour.

C'est Dieu qui appelle, envoie; lui seul a l'initiative et de l'élection et de la mission. Aux premières pages de la Genèse, l'Éternel appelle Abraham et, pour le faire entièrement libre entre ses mains, il le sépare de son clan, puis l'envoie... Cet homme sera le signe vivant de la transcendance divine et toute sa vie s'accomplira dans la foi.

L'Évangile, à son tour, nous rapporte les choix de Dieu : "Jésus les appela; surle-champ, ils laissèrent leur barque et leur père et ils le suivirent. "Même élection, même séparation pour une mission : suivre le Christ, devenir ses témoins au milieu du monde.

Aujourd'hui comme au temps d'Abraham ou des apôtres, l'appel du Seigneur ne cesse de se faire entendre. L'Église nous en révèle le sens : "Il invite à la pratique des conseils évangéliques de façon effective et de propos délibéré, en vue d'une consécration parfaite à Dieu en union à Jésus-Christ Notre-Seigneur. „

Cette vocation spéciale qui est toujours un choix de Dieu, sollicite la réponse de l'homme. Réponse à l'attrait d'une grâce bouleversante ou à peine perceptible parfois, mais réelle, exigeante, qui mène à l'amour et s'accomplit dans la foi.

Cet appel à la perfection des conseils évangéliques se réalise de diverses manières; l'Église reconnaît trois états de perfection : les Instituts religieux, les Sociétés de vie commune, les Instituts séculiers. Il ne faut pas entendre par là que la vie religieuse puisse seule conduire à la perfection. Tout état de vie peut et doit conduire à la sainteté, à condition qu'il apparaisse comme la volonté de Dieu et soit choisi comme tel. Cependant, l'état religieux apporte aux âmes que Dieu y appelle des moyens plus adaptés de tendre à la réalisation de l'amour.

Dans ce numéro, nous nous sommes limitées à l'étude de la vocation religieuse féminine, car elle pose une question à nos contemporains. La littérature profane comme le cinéma ont essayé d'en parler, le plus souvent bien à tort. Ce cahier n'a pas l'ambition de répondre à tous les problèmes qu'elle soulève, mais simplement de donner un sens, une orientation, pour être dans le vrai. Ses divers articles ne peuvent être pris séparément: ils s'éclairent les uns les autres et voudraient du moins montrer que la vocation religieuse a son origine et sa fin dans le mystère de Dieu; seule, une vue de foi peut nous approcher de la vérité.

Marie-Edmond 


\section{Annexe 2-Éditorial d’Échanges, $n^{\circ} 86$, juin 1968.}

TRANSCENDANCE divine, liberté de l'homme se lient de façon mystérieuse et indiscernable dans l'appel et la réponse qui constituent une vocation religieuse.

Parfois la vocation éclate dans une vie, plus fréquemment elle sourd de la conscience devenant à travers le temps et ses épreuves de plus en plus claire. De toute façon, elle réclame l'adhésion de tout l'être. Elle est, en effet, la réponse de l'homme à un appel de Dieu qui invite à lui consacrer sa vie sans partage et dans le même temps à accomplir une mission dans le monde.

La Bible rapporte l'histoire de quelques-uns des choix de Dieu. Dès les premières pages de la Genèse, il est question de la vocation d'Abraham : l'Éternel mande Abraham et, pour le faire entièrement libre entre ses mains, il le sépare de son clan, puis l'envoie. À son tour, l'Évangile raconte comment le Christ choisit ses disciples: "Jésus les appela; sur le champ, ils laissèrent leur barque et ils le suivirent... ». Même élection, même séparation pour une mission : suivre le Christ, devenir ses témoins au milieu du monde.

Aujourd'hui, comme aux différentes étapes de l'histoire des hommes, l'appel de Dieu ne cesse de se faire entendre; des hommes, des femmes quittent leur famille, le monde pour répondre au Seigneur. Réponse à l'attrait d'une grâce bouleversante ou à peine perceptible mais réelle, exigeante qui mène à l'amour et s'accomplit dans la foi.

À quelle vie conduit donc l'appel et tous les chrétiens ne sont-ils pas appelés à vivre l'Évangile? Oui certainement. Il ne faut pas croire que la vie religieuse puisse seule conduire à la perfection de l'amour. Tout état de vie peut et doit conduire à la sainteté, à condition qu'il apparaisse comme la volonté de Dieu et soit choisi comme tel. Cependant, l'état religieux apporte aux personnes que Dieu y appelle des moyens plus adaptés pour elles de tendre à la réalisation de l'amour. L'Église indique le sens spécifique de cet état: "Il invite à la pratique des conseils évangéliques de façon effective et de propos délibéré, en vue d'une consécration parfaite à Dieu en union à Jésus-Christ. »

Il est vrai qu'à travers les siècles cette consécration a pris différentes formes et qu'actuellement la nécessité d'un renouveau, encouragé par le Concile Vatican II, se fait de plus en plus sentir.

Dans un monde en pleine mutation, il est indispensable de retrouver le sens de la vie religieuse. Celle-ci n'est pas une contestation du monde en tant que tel, mais la contestation de ce qui, dans le monde, est contraire à l'Évangile. Elle est, en même temps, le témoignage de l'amour du Christ pour les hommes.

La crise des vocations dont on parle souvent ne peut se comprendre dans le sens d'appels de Dieu qui se feraient plus rares, ni même de réponses moins généreuses. Elle se situe plutôt dans l'inadaptation des formes actuelles en regard des désirs et des capacités de ceux et celles qui aspirent à se consacrer à Dieu.

Une double recherche s'impose, elle est déjà commencée par les religieuses; il est souhaitable qu'elle se fasse aussi par celles qui, appelées à partager leur vie, en récusent les conditions actuelles.

Ce cahier est limité à une étude de la vocation. Un autre y fera suite, dans quelques mois, ayant trait à la crise et au renouvellement de la vie religieuse. Lorsqu'on approfondit cette réalité vivante de la vocation, il importe de garder présent à l'esprit que celle-ci a son origine et sa fin dans le mystère de Dieu et que seule une vue de foi peut nous approcher de la vérité.

Marie-Edmond 


\section{RÉSUMÉ}

La crise des vocations religieuses féminines reconnue plus tardivement que celle des vocations sacerdotales, a contraint l'Église catholique à réfléchir dans les années 1960/1970 sur l'avenir de la vie religieuse. Devant l'échec de la pastorale des vocations pour remédier à la crise du recrutement et afin de faire face à une vague de départs, elle a apporté des réponses ecclésiales en faveur de la modernisation des conditions de vie des religieuses et conduit une réflexion théologique visant à faire des religieuses des actrices de leur vocation. Au cour de ces évolutions collectives, l'itinéraire singulier de Françoise Vandermeersch, Auxiliatrice et directrice de la revue Échanges en fournit un observatoire privilégié.

\section{ABSTRACT}

The crisis of the feminine religious vocations was acknowledged later than that of the priestly vocations, yet it compelled the Roman Catholic Church to reconsider the future of religious life in the 1960s and 1970s. When faced with the failure of the pastoral campaign to arouse vocations and remedy the recruiting predicament but also to cope with a wave of forsaking, the Church gave ecclesial answers so as to modernize the nuns' living conditions and then carried out a theological reflection so that nuns could become more deeply involved in their own vocations. Francoise Vandermeersch who belonged to the Congrégation des Auxiliatrices and was the manageress of the review Échanges gives us the opportunity to have a special look into it thanks to her uncommon progression in the depths of these collective evolutions. 